Banken, bankeigene Kapitalanlage-

gesellschaften und Aktienemissionen

Theodor Baums/Erik Theissen

Arbeitspapier Nr. 66 


\title{
Banken, bankeigene Kapitalanlagegesellschaften und
}

\author{
Aktienemissionen* \\ Theodor Baums / Erik Theissen **
}

\begin{abstract}
Zusammenfassung
Es gibt Überlegungen, Kreditinstituten den Besitz der Anteilsmehrheit an Kapitalanlagegesellschaften (KAGs) zu untersagen. Dahinter steht die Vorstellung, daß solche Beteiligungen Gestaltungsspielräume eröffnen, die mißbräuchlich genutzt werden. Die Neuemission von Aktien ist einer der Fälle, die in diesem Zusammenhang erörtert werden. Ziel dieser Arbeit ist es zu prüfen, ob die zum Konzernverbund einer konsortialführenden Bank gehörenden KAGs bei Erstemissionen anders behandelt werden als andere KAGs.
\end{abstract}

Untersucht werden 46 Neuemissionen der Jahre 1994 bis 1997. Daten über von den Fonds erworbene Aktien wurden den Rechenschafts- und Halbjahresberichten entnommen. Im Durchschnitt erwerben die untersuchten Fonds etwa 7\% der emittierten Aktien. Ein Zusammenhang zwischen der Höhe des Anteilserwerbs und der nachfolgend realisierten Rendite kann nicht nachgewiesen werden. Die der jeweils konsortialführenden Bank zuzuordnenden Fonds erwerben mehr Anteile an einer Neuemission, wenn die ihnen zugeordnete Bank bei der Emission als Konsortialführerin auftritt. Eine Vorhersagbarkeit in dem Sinne, daß der Anteilserwerb in Beziehung zu der nachfolgend realisierten Rendite stünde, kann nicht festgestellt werden. Zudem ergeben sich keine Anhaltspunkte dafür, daß KAGs bei schlechtem Renditeverlauf der Neuemissionen Kurspflegeaufgaben übernehmen.

Insgesamt deuten die Ergebnisse darauf hin, daß die KAGs in ihren Anlageentscheidungen unabhängig sind, und daß keine mißbräuchliche Nutzung eventuell vorhandener Informationsvorsprünge vorliegt. Allerdings ist die Datenbasis, bedingt durch die Vorschriften über die Rechnungslegung der Fonds, unbefriedigend. Es wäre daher zu überlegen, den Fonds stringentere Berichtspflichten aufzuerlegen.

JEL classification: G21, G38

\footnotetext{
* Die Verf. danken der VW-Stiftung für großzügige Unterstützung.

** Prof. Dr. Theodor Baums, Institut für Handels- und Wirtschaftsrecht, Universität Osnabrück, Katharinenstr. 15, D-49069 Osnabrück; Email: tbaums@rz.uni-osnabrueck.de.

Dr. Erik Theissen, Lehrstuhl für Kreditwirtschaft und Finanzierung, Johann Wolfgang Goethe-Universität Frankfurt/Main, Mertonstr. 17-21 (PF 88), D-60054 Frankfurt; Email: theissen@ wiwi.uni-frankfurt.de.
} 


\section{Problemstellung}

Im Zusammenhang mit der generellen Diskussion um die „Macht der Banken“ wird auch die Erbringung einer großen Zahl unterschiedlicher Finanzdienstleistungen durch die deutschen Universalbanken kritisiert. Ein Beispiel für diese Angebotskonzentration ist der Markt für Investmentfonds. Der überwiegende Teil der inländischen Kapitalanlagegesellschaften - gemessen am verwalteten Fondsvolumen annähernd 90\% - befindet sich im Besitz von Kreditinstituten.

Es ist vorgeschlagen worden, Kreditinstituten und Versicherungen die Beteiligung an Kapitalanlagegesellschaften generell zu verbieten. ${ }^{1}$ Hinter derartigen Vorstößen steht die Vorstellung, die Konzentration des Angebots von Fondszertifikaten und anderen Bankdienstleistungen schaffe Gestaltungsspielräume, die mißbräuchlich genutzt würden. Als Ansatzpunkte für solche Aktivitäten werden die Ausübung von Stimmrechten auf Hauptversammlungen, die Umgehung von Meldevorschriften beim Aufbau von Beteiligungen und anlegerschädigendes Verhalten im Zusammenhang mit der Erstemission von Aktien (Initial Public Offerings, IPOs) genannt. BAUMS / KÖNIG (1996) untersuchen die genannten Punkte empirisch. Ihre Ergebnisse lassen die Forderung nach einer generellen Trennung von Banken und Kapitalanlagegesellschaften nicht als gerechtfertigt erscheinen.

Ziel der vorliegenden Arbeit ist eine detaillierte Untersuchung des dritten oben erwähnten Vorwurfs, nämlich der Möglichkeit anlegerschädigenden Verhaltens im Zusammenhang mit Aktienemissionen. Dabei wird auf einen im Vergleich zu der frühen Arbeit von BAUMS / KÖNIG (1996) quantitativ und qualitativ erweiterten Datensatz zurückgegriffen, der die Anwendung verfeinerter Analysemethoden erlaubt.

Grundsätzlich lassen sich drei Ansatzpunkte für anlegerschädigendes Verhalten identifizieren:

1. Bevorzugung bei der Zuteilung: Banken teilen der mit ihnen im Konzernverbund stehenden Kapitalanlagegesellschaft eine relativ größere Zahl von Aktien zu als anderen Zeichnern.

2. Unterbringung ansonsten nicht plazierbarer Aktien: Bei Emissionen mit unzureichendem Zeichnungsvolumen könnten konzernangehörige Kapitalanlagegesellschaften dazu veranlaßt werden, nicht plazierte Aktien in das Vermögen verwalteter Fonds zu übernehmen.

\footnotetext{
1 Die SPD-Bundestagsfraktion hat 1995 einen entsprechenden Vorschlag im Rahmen eines Entwurfs für ein
} „Transparenz- und Wettbewerbsgesetz“ vorgelegt (BT-Drucks. 13/367). 
3. Übertragung von Kurspflegeaufgaben: Konzernangehörige Kapitalanlagegesellschaften könnten dazu veranlaßt werden, bei nicht zufriedenstellender Kursentwicklung Aktien an der Börse zu erwerben, um auf diese Weise den Kurs zu stützen.

Im folgenden werden diese drei Punkte ausführlich erläutert und empirisch testbare Hypothesen formuliert. Anschließend werden die verwendeten Daten und die Untersuchungsmethodik beschrieben. Im Anschluß daran werden die Ergebnisse dargestellt und diskutiert. Eine Zusammenfassung der Resultate beschließt die Arbeit.

\section{Ansatzpunkte für anlegerschädigendes Verhalten und Hypothesen}

Die grundsätzlichen Ansatzpunkte für anlegerschädigendes Verhalten wurden bereits genannt. Im folgenden soll eine ausführlichere Diskussion dieser Punkte erfolgen, die in die Formulierung testbarer Hypothesen mündet.

Die nachstehenden Überlegungen gehen von folgender Ausgangssituation aus. Ein Kreditinstitut ist konsortialführendes Institut bei einer Aktienerstemission und gleichzeitig Anteilseigner einer Kapitalanlagegesellschaft. Das Kreditinstitut hat aufgrund seiner Tätgikeit als Konsortialführer Informationen über den Wert der emittierten Aktien, die bei der Festlegung des Emissionspreises bzw. der Book-Building-Spanne zugrundegelegt werden. Während der Zeichnungsfrist liefert das eingehende Zeichnungsvolumen Informationen über den Erfolg der Emission.

\section{Zeichnung übersteigt verfügbares Emissionsvolumen}

Als erstes sei der Fall betrachtet, daß das Zeichnungsvolumen das verfügbare Emissionsvolumen übersteigt. In diesem Fall muß eine Zuteilung erfolgen. Dabei haben die Konsortialbanken regelmäßig einen diskretionären Spielraum, sie können also entscheiden, wessen Zeichnungswünsche in welchem Umfang berücksichtigt werden. Es ist plausibel, daß bei dieser Entscheidung die Eigeninteressen des Kreditinstituts - etwa die Auswirkungen auf zukünftige Geschäftsabschlüsse - berücksichtigt werden. Dabei kann eine konzernverbundene Kapitalanlagegesellschaft wie folgt berücksichtigt werden:

a) Die konzernverbundene Kapitalanlagegesellschaft wird genauso behandelt wie andere Zeichner. Insbesondere wird sie genauso behandelt wie konzernfremde Kapitalanlagegesellschaften. 
b) Die konzernverbundene Kapitalanlagegesellschaft wird bei der Zuteilung bevorzugt behandelt. Sie erhält eine gemessen an ihrem Zeichnungsvolumen größere Zuteilung als andere Zeichner. Der Anreiz für ein derartiges Verhalten kann darin bestehen, der konzernangehörigen Kapitalanlagegesellschaft auf diese Weise zu einem vorteilhaften Investment und dadurch zu einer guten Performance zu verhelfen. Dem liegt die Vorstellung zugrunde, daß bei einer überzeichneten Emission der erste Börsenkurs über dem Emissionspreis liegen wird, daß also Zeichnungsgewinne wahrscheinlich sind. $\mathrm{Zu}$ fragen ist nun, inwieweit die Bevorzugung der konzernangehörigen Kapitalanlagegesellschaft anlegerschädigend ist. Zunächst ist es denkbar, daß der Entscheidung der Bank Insiderinformationen zugrundeliegen, die sie etwa durch ihre Rolle als Konsortialführerin erhalten hat (vgl. zu dieser Hypothese ADAMS 1996). Jedoch kann auch ohne das Vorliegen von Insiderhandel eine Benachteiligung anderer Marktteilnehmer begründet werden. Die Bank entscheidet über die Zuteilung, nachdem sie anhand der eingehenden Zeichnungsaufträge Informationen über den Erfolg der Emission erhalten hat. Wenn nun die konzerneigene Kapitalanlagegesellschaft nach einem positiven Signal eine höhere Zuteilung erhält, so bedeutet das, daß andere Zeichner bei erfolgreichen Emissionen relativ niedrige, dagegen bei erfolglosen Emissionen hohe Zuteilungen erhalten. Damit ist ein Nachteil verbunden, der dem in der Literatur bekannten Winner's Curse-Phänomen entspricht. ${ }^{2}$ Die Argumentation ähnelt im übrigen der Erklärung, die RocK (1986) für das verbreitete Phänomen des Underpricings von IPOs liefert.

c) Die konzernangehörige Kapitalanlagegesellschaft wird bei der Zuteilung benachteiligt, sie erhält eine gemessen an ihrem Zeichnungsvolumen geringere Zuteilung als andere Zeichner. Ein solches Verhalten, obschon auf den ersten Blick widersinnig, kann durchaus rational sein. Ein Kunde, dessen Zeichnungswunsch nicht berïcksichtigt wird, beobachtet dies unmittelbar und kann daraus Konsequenzen ziehen. Der Fondsanleger dagegen erhält zunächst keine Informationen darüber, inwieweit die Zeichnungswünsche der Kapitalanlagegesellschaft berücksichtigt wurden. Im Falle eines Publikumsfonds lassen nur die Rechenschaftsbzw. Halbjahresberichte erkennen, welche Aktien zum Fondsvermögen gehören. Der genaue Erwerbszeitpunkt ist dort jedoch nicht ersichtlich; der Kunde kann also nicht erkennen, ob die Aktien per Erscheinen oder nachträglich über die Börse erworben wurden. Eine Benachteiligung der eigenen Kapitalanlagegesellschaft wirkt sich möglicherweise negativ auf die Performance aus. Da zwischen Fondsperformance und Anteilsabsatz ein zwar statistisch signifikanter, aber nur schwacher Zusammenhang existiert (vgl. KRAHNEN / SCHMID /

\footnotetext{
2 Vgl. hierzu etwa KAGEL / LEvin (1986).
} 
THEISSEN 1997), kann die Benachteiligung der konzernangehörigen Kapitalanlagegesellschaft mit geringeren Kosten verbunden sein als die anderer Kunden.

In einer empirischen Untersuchung für den durch ein Universalbanksystem mit hohem Konzentrationsgrad gekennzeichneten israelischen Markt finden BER / YAFEH / YOSHA (1998) Hinweise auf eine Benachteiligung von Kapitalanlagegesellschaften. Sie stellen fest, daß Emissionen, die zu einem höheren Anteil von Fonds gezeichnet werden, eine schlechtere Rendite im Jahr nach dem Börsengang aufweisen. Daraus schließen sie, daß die Fonds (und damit letztlich natürlich die Anleger) zu hohe Preise für die Aktien zahlen.

\section{Nicht vollständig gezeichnete Emission}

Als nächstes sei der Fall einer nicht vollständig gezeichneten Emission betrachtet. Die mit dem Emittenten geschlossenen Verträge sehen regelmäßig vor, daß in diesem Fall die Konsortialbanken die nicht gezeichneten Aktien übernehmen. Damit sind Risiken verbunden, zumal auch die nicht vollständige Zeichnung der Emission ein Indiz dafür sein kann, daß der spätere Börsenpreis unter dem Emissionskurs liegen wird. Die Bank kann nun diese Risiken auf die Kunden der konzerneigenen Kapitalanlagegesellschaft abwälzen, indem sie diese zur Zeichnung veranlaßt. Motivation für ein solches Verhalten kann wiederum sein, daß die Kunden der Kapitalanlagegesellschaft dieses Verhalten nicht direkt beobachten und damit auch nicht ohne weiteres sanktionieren können.

\section{3. Übertragung von Kurspflegeaufgaben}

Eine Emission, bei der die Zeichner Verluste erleiden, schmälert die Reputation der konsortialführenden Bank. Daher kann ein Anreiz bestehen, bei nicht zufriedenstellendem Kursverlauf Kurspflege zu betreiben, also Aktien an der Börse zu erwerben. Dabei werden jedoch Aktien erwoben, die die Bank eigentlich nicht in ihr Portfolio aufnehmen wollte. Daraus kann ein Anreiz resultieren, die Aufgabe der Kurspflege der Kapitalanlagegesellschaft zu übertragen. Diese wird dadurch veranlaßt, ein Portfolio zu halten, das nicht ihren Vorstellungen entspricht. Inwieweit dies zu einer geringeren Rendite führt, hängt von der Bewertung am Aktienmarkt ab. Wenn dieser informationseffizient in Bezug auf die der Entscheidung der Bank zugrundeliegenden Informationen ist, wird die Fondsrendite im Erwartungswert durch die Übernahme von 
Kurspflegeaktivitäten nicht sinken; ${ }^{3}$ jedoch kann sich das (unsystematische) Risiko des Fondsportfolios erhöhen. Allerdings ist unter der Annahme der Informationseffizienz die Kurspflege nicht sinnvoll. Die Beobachtung von Kurspflegeaktivitäten impliziert damit, daß die Bank entweder die Informationseffizienz des Marktes in Frage stellt oder aber über Informationen verfügt, die anderen Marktteilnehmern nicht zugänglich sind.

\section{Hypothesen}

Die genannten Verhaltensweisen lassen sich in empirisch testbare Hypothesen umsetzen. Dabei ist, entsprechend der obigen Argumentation, zunächst eine Unterscheidung in erfolgreiche und erfolglose Emissionen erforderlich. Als einfaches Kriterium bietet sich hierfür der Zeichnungsgewinn, also die Differenz zwischen erstem Börsenkurs und Emissionspreis an. Eine Bevorzugung konzernangehöriger Kapitalanlagegesellschaften führt dann zu folgender Hypothese:

H1: Kapitalanlagegesellschaften, die zum Konzern der konsortialführenden Bank gehören, erhalten - aggregiert über alle Publikumsfonds - bei erfolgreichen Emissionen höhere Zuteilungen als andere Kapitalanlagegesellschaften.

Erfolgt die Ungleichbehandlung nur über die Wahl der Zuteilungsquote, so sollte bei erfolglosen Emissionen kein Unterschied zwischen konzerneigenen und fremden Kapitalanlagegesellschaften feststellbar sein. Werden zusätzlich Insiderinformationen bei der Entscheidung berücksichtigt, so läßt sich zusätzlich folgende Hypothese formulieren:

H2: Kapitalanlagegesellschaften, die zum Konzern der konsortialführenden Bank gehören, erhalten - aggregiert über alle Publikumsfonds - bei erfolglosen Emissionen niedrigere Zuteilungen als andere Kapitalanlagegesellschaften, weil sie ein geringeres Volumen gezeichnet haben.

Der Fall einer Benachteiligung der konzerneigenen Kapitalanlagegesellschaft würde zu den entsprechend entgegengesetzten Hypothesen führen. Tatsächlich ist es, wie oben ausgeführt wurde, denkbar, daß die konzerneigene Kapitalanlagegesellschaft im Fall erfolgreicher Emissionen gegenüber anderen Kunden benachteiligt wird. Es ist jedoch nicht plausibel, daß eine solche Benachteiligung gegenüber anderen Kapitalanlagegesellschaften erfolgt. Da mit den verfügbaren Daten aber nur dieser Fall untersucht werden kann, ergibt sich hier keine sinnvolle Hypothese.

\footnotetext{
3 Von den mit der Kurspflege verbundenen Transaktionskosten wird dabei abgesehen.
} 
Anders ist dagegen der Fall einer erfolglosen Emission gelagert. Wenn die konzerneigene Kapitalanlagegesellschaft veranlaßt wird, Aktien zu erwerben, die ansonsten die Bank selbst übernehmen müßte, so ergibt sich:

H3: Kapitalanlagegesellschaften, die zum Konzern der konsortialführenden Bank gehören, erhalten - aggregiert über alle Publikumsfonds - bei erfolglosen Emissionen höhere Zuteilungen als andere Kapitalanlagegesellschaften, weil sie ein höheres Volumen gezeichnet haben.

Schließlich würde die Übertragung von Kurspflegeaufgaben dazu führen, daß die konzerneigene Kapitalanlagegesellschaft im Fall einer ungünstigen Entwicklung des Börsenkurses nach der Emission ihren Aktienbestand erhöht:

H4: Kapitalanlagegesellschaften, die zum Konzern der konsortialführenden Bank gehören, erhöhen - aggregiert über alle Publikumsfonds - bei erfolglosen Emissionen ihren Aktienbestand nach der Emission (absolut oder zumindest relativ zu anderen Kapitalanlagegesellschaften).

Ein direkter Test der angeführten Hypothesen erfordert Daten über die Portfoliostruktur der Fonds, die in dieser Form nicht verfügbar sind. Daher werden im folgenden zunächst die verfügbaren Daten beschrieben. Daran anschließend wird dann erläutert, in welcher Form und mit welcher Methodik ein Test der Hypothesen erfolgen kann.

\section{Daten und Untersuchungsmethodik}

Wertpapierfonds müssen gemäß den Vorschriften des Gesetzes über Kapitalanlagegesellschaften (KAGG) den Anlegern Rechenschaft über Zusammensetzung und Entwicklung des FondsSondervermögens ablegen. § 24 KAGG schreibt die Publikation von entsprechenden jährlichen Rechenschaftsberichten sowie von Halbjahresberichten vor, die im Bundesanzeiger zu veröffentlichen sind. Diese enthalten stichtagsbezogene Aufstellungen der genauen Portfoliozusammensetzung. Zudem ist die Summe der in den vorangegangenen sechs Monaten getätigten Käufe und Verkäufe jeder Wertpapiergattung anzugeben. Diese Angaben erfolgen jedoch aggregiert und insbesondere ohne Angabe des Erwerbs- bzw. Veräußerungszeitpunkts. Damit ist also nicht erkennbar, ob Aktien per Erscheinen oder später an der Börse erworben wurden.

Der Informationsgehalt der Angaben sei anhand der drei in der nachfolgenden Tabelle aufgeführten Fälle erläutert. Die Angaben beziehen sich auf eine Aktie, die in dem Sechsmonatszeitraum, über den berichtet wird, emittiert wurde. 


\begin{tabular}{c|c|c|c} 
Fall & Bestand am Stichtag & Käufe & Verkäufe \\
\hline I & 10.000 & 10.000 & 0 \\
\hline II & 0 & 10.000 & 10.000 \\
\hline III & 5.000 & 10.000 & 5.000
\end{tabular}

Angaben in den Rechenschafts- bzw. Halbjahresberichten

In Fall I hat der Fonds 10.000 Aktien erworben und keine weiteren Transaktionen getätigt. Es ist nicht erkennbar, ob der Erwerb per Emission oder später erfolgte. Es ist zudem nicht erkennbar, ob dem Erwerb eine Transaktion zugrundelag oder ob der Aufbau der Position schrittweise erfolgte. In Fall II ist erkennbar, daß der Fonds zwischen Emissionszeitpunkt und Berichtsstichtag 10.000 Aktien erworben und wieder verkauft hat. Erwerbszeitpunkt und zeitliche Struktur der Käufe bzw. Verkäufe sind nicht erkennbar. Im Fall III wurden insgesamt 10.000 Aktien gekauft und 5.000 verkauft; Datum und zeitliche Struktur der Transaktionen sind wiederum nicht erkennbar. In den Fällen II und III kann keine Aussage über den maximalen Bestand gemacht werden. In Fall II etwa könnten erst 10.000 Aktien gekauft und dann wieder verkauft worden sein (Maximalbestand 10.000), es könnten aber auch zweimal hintereinander 5.000 Aktien jeweils zunächst gekauft und dann wieder verkauft worden sein (Maximalbestand 5.000).

Ein weiteres Problem besteht in den unterschiedlichen Rechnungsjahren der Fonds. Es ist keineswegs immer das Kalenderjahr auch Rechnungsjahr; insbesondere ein Rechnungsjahr vom 1.10. bis zum 30.9. ist weit verbreitet. Daher beziehen sich die für unterschiedliche Fonds verfügbaren Angaben nicht auf den gleichen Stichtag und den gleichen Halbjahreszeitraum.

Diese Datenlage bedeutet, daß ein Test der Hypothesen 1 bis 4 erschwert wird bzw. daß Annahmen über den Zeitpunkt des Aktienerwerbs benötigt werden. Für die im folgenden darzustellenden Untersuchungen wurde angenommen, daß alle Fonds die aufgrund der verfügbaren Angaben maximal mögliche Anzahl an Aktien (in den o.a. Fällen I bis III also jeweils 10.000) per Emission erworben haben. Bei Richtigkeit dieser Annahme haben die unterschiedlichen Rechnungsjahre keine Auswirkungen auf die Ergebnisse. 
Die soeben erläuterte Annahme über den Erwerbszeitpunkt ist nicht unkritisch. Daher ist nach Möglichkeiten zu suchen, ihre Richtigkeit zu überprüfen. ${ }^{4}$ Dies ist auf zweierlei Weise möglich:

1. Bei einigen der untersuchten Emissionen fällt der Tag der ersten Notierung an der Börse in einen anderen Abrechnungszeitraum als der Tag der Emission. Beispielsweise wurde die Aktie Bertrandt AG am 1.10.1996 erstmals notiert, die Emission erfolgte aber bereits im September. Fonds, bei denen das Rechnungsjahr oder die Berichtsperiode für ein Halbjahr am 30.9.1997 endet, bieten daher eine Möglichkeit zur Überprüfung der Annahme über den Erwerbszeitpunkt. Ist sie richtig, sollten die erworbenen Aktien bereits zum 30.9.1997 im Bestand ausgewiesen sein, und es sollte im darauffolgenden Halbjahr keine Bestandserhöhung erfolgen. Ebenfalls in Betracht gezogen werden können Fälle, in denen der Tag der ersten Notiz kurz vor das Ende eines Berichtszeitraums fiel. In dieser Konstellation entfallen nur wenige Börsenhandelstage auf dieses Berichtsintervall. Erfolgt bis zum nächsten Berichtszeitpunkt eine Bestandserhöhung, so deutet dies darauf hin, daß der Bestand langsam durch Erwerb über die Börse aufgebaut wird.

2. Bei der Ermittlung des Inventarwertes eines Fonds, der dem Rücknahmepreis zugrundeliegt, werden neu emittierte Aktien vor der ersten Börsennotiz mit dem Emissionspreis bewertet. $\mathrm{Ab}$ dem ersten Handelstag werden sie dagegen mit dem an der Börse festgestellten Kurs bewertet. Demzufolge schlägt sich ein positiver oder negativer Zeichnungsgewinn am ersten Börsenhandelstag in der Fondsrendite nieder. Bei Emissionen mit einer hohen Zeichnungsrendite, also einem ersten Börsenkurs, der deutlich über dem Emissionspreis liegt, sollte sich also bei denjenigen Fonds, die die Aktie per Emission erworben haben, am Tag der ersten Börsennotiz eine überdurchschnittliche Rendite zeigen. Dieser Zusammenhang kann prinzipiell genutzt werden, um die Richtigkeit der Annahme über den Erwerbszeitpunkt zu überprüfen.

Eine Überprüfung anhand der erstgenannten Konstellation ergab ein uneinheitliches Bild. Insgesamt 41 Fälle, die sich auf 8 Emissionen bezogen, konnten einbezogen werden. ${ }^{5}$ Dabei ergab

4 Eine solche Überprüfung ist auch deshalb angebracht, weil Fondsmanagern oft sogenanntes „Window Dressing“ nachgesagt wird. Danach werden kurz vor Abschluß eines Rechnungsjahres bzw. Berichtszeitraums solche Aktien gekauft, die zuvor eine gute Performance gezeigt haben. Auf diese Weise soll den Adressaten der Rechenschaftsberichte, die ja den genauen Erwerbszeitpunkt nicht kennen, suggeriert werden, das Fondsmanagement habe die betreffenden Aktien frühzeitig erworben. Sofern derartiges Window Dressing auch bei erfolgreichen Neuemissionen durchgeführt wird, könnte dies die Ergebnisse der vorliegenden Arbeit beeinflussen.

5 Ein „Fall“ ist ein Fonds, dessen Berichtsintervall nicht mehr als eine Woche nach dem ersten Handelstag der Emission endet und der im Berichtsintervall, in dem die Emission stattfand, oder im darauffolgenden Berichtsintervall die betreffende Aktie gekauft hat. 
sich in 28 Fällen $(68,3 \%)$ ein Bild, das mit der Annahme über einen Erwerb per Emission vereinbar ist; in den restlichen Fällen kam es dagegen im Berichtsintervall nach der Emission zu Bestandserhöhungen.

Die Überprüfung anhand des zweiten Verfahrens wurde auf Basis der Emissionen mit einer Zeichnungsrendite von mindestens 5\% durchgeführt. Auch hier ergab sich kein einheitliches Bild. Fonds, die die betreffende Aktie am ersten Berichtszeitpunkt nach der Emission im Bestand ausweisen, verzeichnen am Tag der ersten Börsennotiz zwar im Durchschnitt über alle Emissionen eine höhere Rendite als die Gesamtheit aller Fonds, ${ }^{6}$ jedoch beträgt diese Differenz im Durchschnitt nur 0,03\%, und es gibt bei einer Reihe von Emissionen auch negative Werte. ${ }^{7}$

Insgesamt kann also von einer Bestätigung der Richtigkeit der Annahme über den Erwerbszeitraum nicht gesprochen werden. Dies ist bei der Durchführung der Untersuchung und der Interpretation der Ergebnisse zu berücksichtigen. Wie im weiteren Verlauf noch auszuführen ist, sind allerdings die wesentlichen Schlußfolgerungen dieser Arbeit von einer möglichen Verletzung der Annahme über den Erwerbszeitpunkt nicht betroffen.

In die Untersuchung wurden alle Aktien einbezogen, die zwischen dem 1.1.1994 und dem 30.6.1997 erstmals angeboten und anschließend in den amtlichen Handel, den Handel am geregelten Markt oder den Handel am Neuen Markt einbezogen wurden. Dies ergibt insgesamt 46 Emissionen. Für diese wurden jeweils der Emissionspreis, der Schlußkurs ${ }^{8}$ des ersten Handelstages sowie die Monatsschlußkurse der nachfolgenden zwölf Monate ermittelt. Die folgende Übersicht zeigt die realisierten Zeichnungsrenditen. Bemerkenswert ist, daß bei keiner der 46 untersuchten Emissionen eine negative Zeichnungsrendite auftrat. Für die vorliegende Untersuchung ist dies insofern unglücklich, als die für den Fall negativer Zeichnungsrenditen formulierten Hypothesen nicht überprüft werden können. ${ }^{9}$

6 Diese Berechnung wurden nur für diejenigen Fonds durchgeführt, deren Anlageschwerpunkt deutsche Aktien sind. Eine Einbeziehung gemischter und international investierender Fonds ist aufgrund der fehlenden Vergleichbarkeit der Renditen unzweckmäßig.

7 Einer der Gründe für dieses Resultat ist die Tatsache, daß die aus einer Neuemission erworbenen Aktien regelmäßig nur einen sehr kleinen Teil des Fondsvolumens ausmachen (im Durchschnitt etwas mehr als $1 \%$, errechnet unter Zugrundelegung der Annahme über den Erwerbszeitpunkt). Bezogen auf das Fondsvolumen leistet eine Emissionsrendite von 5\% also einen Renditebeitrag von 0,05\%. Dieser Wert ist sehr klein im Verhältnis zu den Renditeunterschieden, die an einem gegebenen Tag zwischen den verschiedenen Fonds bestehen. Selbst wenn man nur die einander relativ ,ähnlichen“ Fonds mit Anlageschwerpunkt in deutschen Aktien (gemäß der Klassifikation des BVI) betrachtet, beträgt die Standardabweichung der Renditen im Durchschnitt 0,37\%.

8 Sofern eine Aktie an mehr als einer Börse notiert war, wurde der Kurs der Frankfurter Wertpapierbörse gewählt.

9 Eine Ausweitung des Untersuchungszeitraums beseitigt diesen Mangel nicht. Auch in den Jahren 1991 bis 1994 gab es nur eine Emission (Aachener und Münchener Leben), die eine negative Zeichnungsrendite 


\begin{tabular}{c|c|c|c|c|c|c}
$\begin{array}{c}\text { Zeichnungs- } \\
\text { rendite }\end{array}$ & 0 & $\begin{array}{c}0 \text { bis unter } \\
1 \%\end{array}$ & $\begin{array}{c}1 \% \text { bis unter } \\
2,5 \%\end{array}$ & $\begin{array}{c}2,5 \% \text { bis } \\
\text { unter } 5 \%\end{array}$ & $\begin{array}{c}5 \% \text { bis unter } \\
10 \%\end{array}$ & $\begin{array}{c}10 \% \text { und } \\
\text { darüber }\end{array}$ \\
\hline Anzahl & 5 & 4 & 7 & 5 & 15 & 10
\end{tabular}

\section{Verteilung der Zeichnungsrenditen}

Neben der Zeichnungsrendite der Neuemissionen interessiert ihre weitere Kursentwicklung, ihre „Long-run-Performance“. Sie wird beurteilt anhand der Kursentwicklung vom Tag der ersten Börsennotierung bis zum Monatsschlußkurs des sechsten Monats nach der Erstnotiz (gekennzeichnet als 6+) bzw. des zwölften Monats nach der Erstnotiz (12+). Eine Bereinigung um die Gesamtmarktentwicklung wurde durchgeführt, indem neben der Rendite auch die gegenüber dem CDAX-Performanceindex gemessene Überrendite errechnet wurde. Die nachfolgende Tabelle faßt die Resultate zusammen.

Es ist zu erkennen, daß viele der Werte entweder sehr hohe oder sehr niedrige Sechs- und Zwölfmonatsrenditen aufweisen. Bei Betrachtung der Überrenditen überwiegt die negative Kursentwicklung. Diese Beobachtung ist konsistent mit der in der Literatur diskutierten „Long-run-Underperformance“ von Neuemissionen.

\begin{tabular}{c|c|c|c|c|c|c}
$\begin{array}{c}\text { Performance } \\
\text { in Prozent }\end{array}$ & $<-10$ & $-5--10$ & $-5-0$ & $0-5$ & $5-10$ & $>10$ \\
\hline $\begin{array}{c}\text { Rendite 6+ } \\
\text { Monate }\end{array}$ & 14 & 1 & 6 & 5 & 5 & 14 \\
\hline $\begin{array}{c}\text { Rendite 12+ } \\
\text { Monate }\end{array}$ & 13 & 3 & 5 & 1 & 4 & 15 \\
\hline $\begin{array}{c}\text { Überrendite } \\
\text { 6+ Monate }\end{array}$ & 18 & 8 & 3 & 2 & 2 & 12 \\
\hline $\begin{array}{c}\text { Überrendite } \\
\text { 12+ Monate }\end{array}$ & 23 & 4 & 2 & 1 & 2 & 9
\end{tabular}

\section{Verteilung der Long-run-Performance}

Nach dieser Charakterisierung der in der Stichprobe enthaltenen Aktien sind nun noch die Fondsdaten zu beschreiben. Einbezogen wurden alle zum öffentlichen Vertrieb zugelassenen Publikumsfonds, die von Kapitalanlagegesellschaften aufgelegt wurden, die Mitglied des Bun- 
desverbandes Deutscher Investment-Gesellschaften eV (BVI) sind. In die Untersuchung nicht einbezogen sind damit Publikumsfonds mit nichtöffentlichem Vertrieb, Spezialfonds und Fonds, die von ausländischen (insbesondere Luxemburger) Tochtergesellschaften deutscher Kapitalanlagegesellschaften aufgelegt wurden. Die Beschränkung der Untersuchung auf Publikumsfonds ist sinnvoll, da die Möglichkeit einer mißbräuchlichen Nutzung von Gestaltungsspielräumen hier plausibler ist als im Fall der stets nur von einem oder wenigen großen Investoren gehaltenen Spezialfonds.

Für die in die Untersuchung einbezogenen Fonds wurden aus den Rechenschafts- und Halbjahresberichten des Zeitraums 1.1.1994 bis 31.121997 die Bestände, Käufe und Verkäufe der zu untersuchenden Aktien ermittelt. Dabei wurden neben den Angaben für den Berichtszeitraum der Emission auch die Angaben aus den beiden nachfolgenden Berichtszeiträumen erhoben. Dadurch können spätere Bestandsveränderungen erfaßt werden, was insbesondere für den Test der die Kurspflege betreffenden Hypothese erforderlich ist. Daten über die Rücknahmepreise aller Publikumsfonds wurden uns von der Vereinigte Wirtschaftsdienste GmbH (vwd) zur Verfügung gestellt.

\section{Ergebnisse}

Im folgenden werden die Ergebnisse in drei Abschnitten dargestellt. Der erste Abschnitt untersucht, wie hoch die von den untersuchten Publikumsfonds insgesamt gezeichneten Anteile an den Neuemissionen sind. Außerdem wird untersucht, ob die Fonds eine Prognosefähigkeit dergestalt besitzen, daß sie höhere Anteile an solchen Emissionen zeichnen, bei denen die Zeichnungsrendite oder die Long-run-Performance positiv ist. Anschließend wird untersucht, ob eine Bevorzugung konzerneigener Kapitalanlagegesellschaften bei der Zuteilung nachgewiesen werden kann. Auch hier wird zusätzlich geprüft, ob der konsortialführenden Bank zugeordnete Fonds eine besondere Prognosefähigkeit besitzen und dementsprechend bei erfolgreichen Emissionen höhere Anteile erwerben. Schließlich wird im dritten Abschnitt untersucht, ob in den Portfolios der Fonds Bestandsveränderungen nachgewiesen werden können, die auf eine Übernahme von Kurspflegeaktivitäten hindeuten.

\section{Zeichnungsverhalten der Fonds}


Eine erste interessante Frage ist die, welchen Anteil an einer Neuemission die in dieser Arbeit betrachteten Publikumsfonds insgesamt zeichnen. Abbildung 1 zeigt ein Histogramm der Zeichnungsanteile. Im Durchschnitt übernehmen die untersuchten Fonds 7,12\% des Emissionsvolumens; der Median liegt bei 5,03\%. ${ }^{10}$ Die Streuung ist erheblich; bei zwei Emissionen hat keiner der Fonds Aktien erworben, beim Börsengang der Tarkett AG haben die Fonds mit 48,2\% nahezu die Hälfte des Emissionsvolumens übernommen.

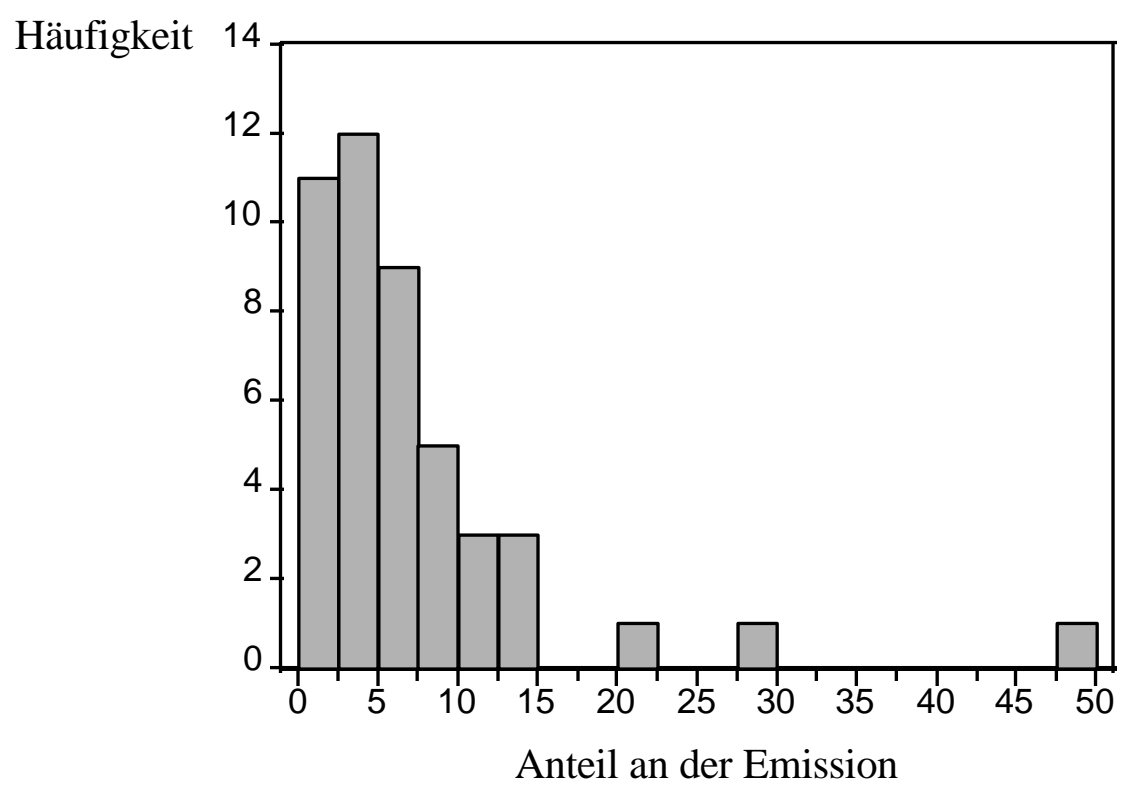

\section{Abbildung 1: Von den untersuchten Fonds übernommene Anteile an den 46 Emissionen}

Eine weitere Frage von Interesse ist die, ob die Fondsmanager bei ihren Zeichnungsentscheidungen in der Lage sind, den Erfolg der Emission zu prognostizieren. Wäre dies der Fall, so wäre zu erwarten, daß die Fonds in ihrer Gesamtheit dann größere Anteile an einer Emission zeichnen, wenn diese Emission sich im Nachhinein als erfolgreich erweist. Der Erfolg kann dabei kurzfristig anhand der Zeichnungsrendite oder längerfristig anhand der Rendite über die nachfolgenden sechs oder zwölf Monate gemessen werden. Bei dieser längerfristigen Betrachtung erscheint es angebracht, neben der einfachen Rendite auch die Überrendite im Vergleich zu einem geeigneten Index (hier: dem CDAX-Performanceindex) zu verwenden. Die folgende Tabelle zeigt die Korrelation zwischen dem von den Fonds übernommenen Anteil an der Emission und dem so berechneten Erfolg der Emission. Die in allen Fällen negative (und nicht statistisch signifikant von Null verschiedene) Korrelation zeigt, daß es keine Anhaltspunkte für eine

10 Es sei daran erinnert, daß die Berechnung auf Basis der ausführlich diskutierten Annahme über den Erwerbszeitpunkt erfolgt. Insofern stellen die hier angegebenen Zahlenwerte eine obere Grenze für die tatsächlichen Werte dar. 
Prognosefähigkeit der Fondsmanager gibt. ${ }^{11}$ Andererseits gibt es aber auch keine Anzeichen dafür, daß Fonds höhere Anteile an solchen Emissionen erwerben, die sich im nachhinein als erfolglos erweisen. Für den israelischen Markt hatten BER / YAFEH / YOSHA (1998) einen solchen Zusammenhang gefunden.

\begin{tabular}{c|c|c|c|c|c} 
Rendite & $\begin{array}{c}\text { Zeichnungs- } \\
\text { rendite }\end{array}$ & $\begin{array}{c}\text { Rendite 6+ } \\
\text { Monate }\end{array}$ & $\begin{array}{c}\text { Rendite 12+ } \\
\text { Monate }\end{array}$ & $\begin{array}{c}\text { Überrendite 6+ } \\
\text { Monate }\end{array}$ & $\begin{array}{c}\text { Überrendite 12+ } \\
\text { Monate }\end{array}$ \\
\hline Korrelation & $-0,06$ & $-0,04$ & $-0,11$ & $-0,01$ & $-0,11$
\end{tabular}

Korrelation zwischen Anteilserwerb und Emissionserfolg

\section{Bevorzugung oder Benachteiligung "eigener" Fonds?}

Die bislang dargestellten Resultate bezogen sich jeweils auf die Gesamtheit aller untersuchten Fonds. Die eingangs dieser Arbeit formulierten Hypothesen bezogen sich dagegen nur auf Fonds, die in einer besonderen Beziehung zur konsortialführenden Bank stehen. Diese Bank hat einerseits mehr Informationen über die Emission, die möglicherweise Eingang in die Anlageentscheidungen des Fondsmanagements finden können, andererseits ist aus den bereits dargelegten Gründen aber auch eine Benachteiligung der eigenen Kapitalanlagegesellschaft denkbar.

Bevor eine Überprüfung vorgenommen werden kann ist zunächst zu klären, welche Kapitalanlagegesellschaften bzw. welche Fonds einer konsortialführenden Bank zugeordnet werden. Für diese Zuordnung wurden folgende Kriterien verwendet:

- Eine Zuordnung erfolgt, wenn die Bank selbst eine Mehrheitsbeteiligung an der Kapitalanlagegesellschaft hält,

- wenn die Kapitalanlagegesellschaft zum gleichen Konzern wie die Bank gehört,

11 Das spricht im übrigen auch dagegen, daß in größerem Umfang Window Dressing betrieben wird. Dies würde nämlich dazu führen, daß Fonds nach der Emission Titel mit einer guten Performance über die Börse erwerben. Da diese Käufe aufgrund der hier gemachten Annahme über den Erwerbszeitpunkt dem bei der Emission übernommenen Anteil zugeschlagen würden, müßte Window Dressing zu einer positiven Korrelation zwischen erworbenem Anteil und nachfolgend realisierter Rendite führen. Dies ist aber, wie die Ergebnisse zeigen, nicht der Fall. 
- sowie bei den dann noch verbleibenden Kapitalanlagegesellschaften, wenn die Bank Depotbank eines Fonds ist. ${ }^{12}$

Die ADIG Allgemeine Deutsche Investmentgesellschaft mbH stellt insofern einen Sonderfall dar, als sowohl die Commerzbank als auch die Bayerische Vereinsbank maßgebliche Anteilseigner dieser Kapitalanlagegesellschaft sind. Die Zuordnung wurde hier dergestalt vorgenommen, daß die Fonds, bei denen die Commerzbank [Bayerische Vereinsbank] Depotbank ist, der Commerzbank [Bayerischen Vereinsbank] zugeordnet wurden. Es verbleiben einige Kapitalanlagegesellschaften, die nicht zugeordnet werden. Es handelt sich um Gesellschaften, deren Anteilseignerbanken und / oder Depotbanken bei keiner der 46 untersuchten Neuemissionen als konsortialführendes Institut auftraten.

Auf Basis dieser Zuordnung wurde nun untersucht, ob die einer konsortialführenden Bank zugeordneten Fonds systematisch höhere oder niedrigere Anteile bei den von der jeweiligen Bank betreuten Emissionen erwerben. Dazu wurde wie folgt vorgegangen. Alle einer Bank zugeordneten Fonds wurden zusammengefaßt. Für jede der 46 Emissionen wurde ermittelt, wieviel Prozent des Emissionsvolumens die Fondsgruppen jeweils erwarben. Schließlich wurden für jede Fondsgruppe zwei Durchschnittswerte ermittelt: Der durchschnittliche Anteilserwerb bei den Emissionen der zugeordneten Bank und der durchschnittliche Anteilserwerb bei allen Emissionen. Die Ergebnisse sind in der folgenden Tabelle dargestellt. ${ }^{13}$

12 Viele der in diese letztere Kategorie fallenden Kapitalanlagegesellschaften sind Tochtergesellschaften von Versicherungen. Darunter gibt es mehrere Fälle, in denen eine Kapitalbeteiligung zwischen der Versicherung und der betreffenden Bank besteht.

13 Es ist zu beachten, daß die Zahlen für unterschiedliche Fondsgruppen nicht vergleichbar sind, weil nicht für das Gesamtvolumen der Fonds kontrolliert wird. 


\begin{tabular}{c|c|c|c} 
Konsortialbank & Anteil alle Emissionen & Anteil eigene Emissionen & $\mathrm{n}$ \\
\hline Baden-Württemb. Bank & 0,062 & 0,38 & 1 \\
\hline Bankges. Berlin & 0,07 & 1,951 & 3 \\
\hline Bayer. Vereinsbank & 0,443 & 0,852 & 4 \\
\hline Commerzbank & 1,332 & 1,904 & 4 \\
\hline Deutsche Bank & 1,4 & 4,773 & 10 \\
\hline $\begin{array}{c}\text { Deutsche Genossen- } \\
\text { schaftsbank }\end{array}$ & 0,093 & 0,306 & 3 \\
\hline Dresdner Bank & 0,74 & 1,516 & 1 \\
\hline Bayr. Hypotheken- u. & 0,117 & 0,586 & 9 \\
\hline Wechselbank & 1,178 & 1,849 & 10,222 \\
\hline Sparkassensektor & 0,02 & nicht definiert & \\
\hline Trinkaus & 1,665 & & \\
\hline Rest & & & \\
\hline
\end{tabular}

\section{Anteilserwerb und Konsortialführerschaft}

Für alle zehn einer Bank zugeordneten Fondsgruppen ergibt sich, daß bei Emissionen der betreffenden Bank im Durchschnitt ein höherer Anteil des Emissionsvolumens erworben wird als im Durchschnitt aller Emissionen. Dafür kann es zwei Gründe geben. Entweder zeichnen die Fondsmanager bei Emissionen, bei denen die eigene „Mutterbank“ Konsortialführerin ist, höhere Anteile als bei anderen Emissionen, oder sie erhalten eine höhere Zuteilungsquote. Nur im zweiten Fall kann von einer Bevorzugung gesprochen werden. Mit den verfügbaren Daten kann nicht zwischen den beiden Fällen unterschieden werden, da nicht beobachtbar ist, wieviele Aktien das Fondsmanagement ursprünglich gezeichnet hat.

Die konsortialführende Bank wird regelmäßig über Informationen über das an die Börse gebrachte Unternehmen verfügen, die anderen Anlegern (und anderen Banken) nicht zur Verfügung stehen. Werden diese Informationen im Interesse der der Bank zugeordneten Fonds genutzt, so sollte sich eine Prognosefähigkeit dergestalt zeigen, daß diese Fonds bei Emissionen, die sich im Nachhinein als erfolgreich erweisen, besonders hohe Anteile zeichnen.

Eine Überprüfung dieser Hypothese ist, wie in Abschnitt IV.1. bereits für die Gesamtheit aller Fonds vorgenommen, möglich, indem die Korrelation zwischen erworbenem Anteil und einem Maß für den Emissionserfolg ermittelt wird. Da die Ermittlung der Korrelation eine ausrei- 
chende Zahl von Beobachtungen voraussetzt, konnte diese Berechnung nur für Emissionen der Deutschen Bank (12 Beobachtungen), der Dresdner Bank (10) und des Sparkassensektors (9 Beobachtungen, davon 8 mit der WestLB als konsortialführendem Institut) durchgeführt werden. Neben dem Korrelationskoeffizienten (oberer Eintrag in den Feldern der nachfolgenden Tabelle) wurde jeweils auch der Rangkorrelationskoeffizient (unterer Eintrag) ermittelt.

Eine hohe Korrelation zwischen erworbenem Anteil und Erfolg der Emission ist allein noch kein Beleg dafür, daß ein Informationsvorsprung mißbräuchlich genutzt wurde. Es kann nämlich nicht ausgeschlossen werden, daß andere Anleger die gleichen Informationen und damit die gleiche Prognosefähigkeit besaßen. Daher wurde jeweils auch ein Korrelationskoeffizient für die Vergleichsgruppe aller Fonds (also nicht nur die dem konsortialführenden Institut zugeordneten) gebildet. Wenn sich für die zugeordneten Fonds, nicht aber für die Vergleichsgruppe eine hohe Korrelation zwischen erworbenem Anteil und Emissionserfolg zeigt, ist der Verdacht der Nutzung von Informationsvorsprüngen gerechtfertigt. Die folgende Tabelle zeigt die Ergebnisse.

\begin{tabular}{c|c|c|c|c|c}
\multirow{2}{*}{ Institut } & \multirow{2}{*}{ Zeichnung } & \multicolumn{2}{|c|}{$6+$ Monate } & \multicolumn{2}{c}{$12+$ Monate } \\
\cline { 3 - 6 } & $-0,139$ & Rendite & Überrend. & Rendite & Überrend. \\
\hline \multirow{2}{*}{ Deutsche Bank } & $-0,198$ & 0,154 & 0,249 & $-0,390$ & $-0,323$ \\
& $-0,170$ & 0,229 & 0,415 & $-0,332$ & $-0,217$ \\
\hline \multirow{2}{*}{ Vergleichswert } & $-0,021$ & 0,154 & 0,267 & $-0,342$ & $-0,271$ \\
& $-0,013$ & 0,350 & 0,469 & $-0,236$ & $-0,091$ \\
\hline \multirow{2}{*}{ Dresdner Bank } & 0,236 & 0,226 & 0,104 & 0,127 & $-0,005$ \\
& 0,046 & 0,612 & 0,479 & 0,25 & 0,083 \\
\hline \multirow{2}{*}{ Vergleichswert } & $-0,273$ & 0,209 & 0,078 & 0,109 & $-0,033$ \\
& $-0,118$ & 0,455 & 0,358 & 0,583 & 0,367 \\
\hline Sparkassensektor & 0,327 & 0,163 & 0,313 & 0,490 & 0,475 \\
& 0,258 & 0,267 & 0,327 & 0,221 & 0,295 \\
\hline Vergleichswert & 0,600 & 0,379 & 0,471 & 0,478 & 0,437 \\
& & 0,633 & 0,617 & 0,238 & 0,429
\end{tabular}

\section{Anteilerwerb bei Konsortialführerschaft und Emissionserfolg}

Viele der Korrelationskoeffizienten liegen nahe bei Null oder sind negativ. Dort, wo positive Korrelationskoeffizienten ermittelt wurden, liegen die Werte für die Vergleichsgruppe in ähnlicher Höhe wie die für die der konsortialführenden Bank zugeordnete Gruppe. Die Ergebnisse 
deuten somit nicht darauf hin, daß es zu einer mißbräuchlichen Nutzung von Informationen kommt.

Es gibt einen weiteren Weg zu testen, ob der konsortialführenden Bank zugeordnete Fonds bei der Zuteilung von Neuemissionen bevorzugt werden. Existiert eine solche Bevorzugung, sollte sich bei diesen Fonds am Tag der ersten Börsennotiz einer Neuemission mit hoher Zeichnungsrendite eine hohe Tagesrendite zeigen. Auf Basis der Rücknahmepreise für die Fonds wurde überprüft, inwieweit dies der Fall ist. Um eine Vergleichbarkeit zwischen den Renditen unterschiedlicher Fonds zu gewährleisten, wurden dazu nur Fonds mit Anlageschwerpunkt in deutschen Aktien herangezogen. Es wurden 24 Neuemissionen mit einer Zeichnungsrendite von mehr als 5\% untersucht. Aus den Fondsrenditen am ersten Notierungstag dieser Unternehmen wurden zwei Durchschnitte errechnet; der Durchschnitt aller Fonds ${ }^{14}$ und der Durchschnitt derjenigen Fonds, die der jeweiligen konsortialführenden Bank zugeordnet wurden. Der Vergleich dieser Renditen ist auch deswegen eine sinnvolle Ergänzung zu den bisher durchgeführten Untersuchungen, weil er unabhängig von der Richtigkeit der Annahme über den Erwerbszeitpunkt ist.

Die Durchschnittsrendite der der jeweiligen konsortialführenden Bank zugeordneten Fonds ist in 12 Fällen geringer als die Durchschnittsrendite aller Fonds. Auch im Mittelwert über alle untersuchten Emissionen ergibt sich eine um 0,017\% niedrigere Rendite der der Konsortialführerin zugeordneten Fonds. Auf Basis einer Untersuchung der Fondsrenditen ergeben sich also keine Anhaltspunkte dafür, daß die konsortialführende Bank die ihr zugeordneten Fonds besser behandelt als andere Fonds. ${ }^{15}$ Auch für eine Schlechterstellung ergeben sich keine Anhaltspunkte, da die berichtete Renditedifferenz zwar negativ, aber nicht statistisch signifikant verschieden von Null ist.

14 Die Zahl der Fonds schwankt dabei zwischen 49 und 55. Die Schwankungen sind zum einen dadurch erklärbar, daß einige Fonds während des Untersuchungszeitraums neu aufgelegt wurden; zum anderen dadurch, daß in einer geringen Zahl von Fällen Rücknahmepreise für einzelne Fonds an einzelnen Tagen nicht verfügbar waren. Vier Fonds konnten in der Datenbasis nicht identifiziert werden; zwei weitere Fonds mußten ausgeschlossen werden, weil die Rücknahmepreise nur in vollen DM enthalten waren, was zu einer Überschätzung der (absoluten) Renditen führt.

15 Diese Aussage steht in einem gewissen Widerspruch zu dem zuvor berichteten Ergebnis, daß Fonds dann einen höheren Anteil am Emissionsvolumen erwerben, wenn die ihnen zugeordnete Bank Konsortialführerin ist. Da nämlich die Zeichnungsrendite in allen untersuchten Fällen positiv ist, müßte sich dies in den Renditen wiederspiegeln. Es gibt zwei mögliche Erklärungen für diesen Widerspruch. Erstens ist denkbar, daß die der konsortialführenden Bank zugeordneten Fonds ihre Anteile an Neuemissionen in stärkerem Maße an der Börse erwerben (und daher nicht von der Zeichnungsrendite profitieren) als andere Fonds. 


\section{3. Übernahme von Kurspflegeaktivitäten}

Wenn Kapitalanlagegesellschaften - freiwillig oder auf Drängen der Anteilseigner-Bank Kurspflegeaktivitäten bei wenig erfolgreichen Emissionen durchführen, dann sollte beobachtbar sein, daß sich bei diesen Emissionen die Bestände der der konsortialführenden Bank zugeordneten Fonds erhöhen. Dies wurde anhand einer Untersuchung von 10 Emissionen mit besonders negativer Kursentwicklung überprüft. Ausgewählt wurden diejenigen zehn Emissionen, bei denen sowohl die 6-Monats- als auch die Jahresrendite weniger als $-10 \%$ betrug.

Nur in drei dieser zehn Fälle kam es zu Bestandserhöhungen bei den der konsortialführenden Bank zugeordneten Fonds. In allen drei Fällen konnte jedoch beobachtet werden, daß andere Fonds ihre Bestände der betreffenden Aktie ebenfalls, und zwar in teilweise deutlich stärkerem Maße, erhöhten. Insofern liefern die Daten keinerlei Anhaltspunkte dafür, daß Kapitalanlagegesellschaften Kurspflegeaufgaben übernehmen.

\section{Zusammenfassung}

Ziel dieser Arbeit war es, anhand einer empirischen Untersuchung zu prüfen, ob die mit einer konsortialführenden Bank konzernverbundenen Kapitalanlagegesellschaften bei Erstemissionen anders behandelt werden als andere Kapitalanlagegesellschaften. Die dazu angestellten Vorüberlegungen zeigten, daß sowohl der Fall einer Bevorzugung als auch der Fall einer Benachteiligung denkbar ist.

In die Untersuchung wurden 46 Neuemissionen des Zeitraums 1.1.1994 bis 30.6.1997 einbezogen. Daten über von den Fonds erworbene Aktien wurden den Rechenschafts- und Halbjahresberichten aller zum öffentlichen Vertrieb zugelassenen Publikumsfonds deutscher Kapitalanlagegesellschaften entnommen. Die Durchführung der Untersuchung wurde durch die Qualität des verfügbaren Datenmaterials beeinträchtigt. Insbesondere war eine Annahme darüber erforderlich, wann die in den Rechenschafts- und Halbjahresberichten dokumentierten Käufe erfolgten.

Im Durchschnitt erwarben die untersuchten Fonds 7,12\% der emittierten Aktien. Ein Zusammenhang zwischen der Höhe des Anteilserwerbs und der Zeichnungsrendite oder der Rendite über die nachfolgenden 6 bzw. 12 Monate konnte nicht nachgewiesen werden.

Zweitens ist es analog der Argumentation in Fußnote 7 denkbar, daß die Auswirkungen der Zeichnungsgewinne auf die Fondsrenditen so gering sind, daß sie von anderen Faktoren überlagert werden. 
Für den nächsten Untersuchungsschritt wurde eine Zuordnung der Fonds zu den Emissionsbanken vorgenommen, wobei Anteilsbesitz an der Kapitalanlagegesellschaft und die Depotbankeigenschaft als Kriterien verwendet wurden. Es konnte gezeigt werden, daß die Fonds mehr Anteile an einer Neuemission erwerben, wenn die ihnen zugeordnete Bank bei der Emission als Konsortialführerin auftritt. Auch hier konnte allerdings keine Voraussagbarkeit in dem Sinne festgestellt werden, daß der Anteilserwerb in Beziehung zu der nachfolgend realisierten Rendite stünde. Zudem ergaben sich keine Anhaltspunkte dafür, daß Kapitalanlagegesellschaften bei schlechtem Renditeverlauf der Neuemissionen Kurspflegeaufgaben übernehmen.

Insgesamt ist damit der höhere Anteilserwerb bei Emissionen der den Fonds zugeordneten Emissionsbank der einzige Beleg für eine Ungleichbehandlung. Es kann jedoch auf Basis des verfügbaren Datenmaterials nicht entschieden werden, ob dies auf ein höheres Zeichnungsvolumen zurückzuführen ist - was unbedenklich wäre, da, wie gesehen, kein Zusammenhang zwischen Anteilserwerb und nachfolgender Rendite besteht, ein Anhaltspunkt für das Ausnutzen eventuell vorhandener Insiderinformationen mithin nicht vorliegt - oder ob dies darauf zurückzuführen ist, daß die der konsortialführenden Bank zugeordneten Fonds bei gleichem Zeichnungsvolumen mehr Anteile zugeteilt bekommen. Letzteres wäre, wie die Argumentation in Abschnitt II gezeigt hat, grundsätzlich nicht unbedenklich. Allerdings konnte eine Auswirkung des Effektes auf die Fondsrenditen nicht nachgewiesen werden. Insofern finden sich keine Anhaltspunkte für anlegerschädigendes Verhalten.

Insgesamt deuten die Ergebnisse der vorliegenden Untersuchung somit darauf hin, daß die Kapitalanlagegesellschaften in ihren Anlageentscheidungen unabhängig sind, und daß eventuell vorhandene Informationsvorsprünge nicht mißbräuchlich ausgenutzt werden. Allerdings ist die Datenbasis, bedingt durch die Vorschriften über die Rechnungslegung der Fonds, unbefriedigend. Es wäre daher zu überlegen, den Fonds stringentere Berichtspflichten aufzuerlegen. $\mathrm{Zu}$ denken wäre dabei etwa an ergänzende Angaben über Transaktionszeitpunkte und -preise. 


\section{Literaturverzeichnis}

ADAMS, MichaEl (1996): Bankenmacht und Deutscher Juristentag. Zeitschrift für Wirtschaftsrecht 37-38, S. 1590-1602.

BAUMS, THEODOR / KÖNIG, MARKUS (1996): Universalbanken und Investmentfonds: Aktuelle Reformfragen. Arbeitspapier Nr. 13/96, Institut für Handels- und Wirtschaftsrecht, Universität Osnabrück.

Ber, Hedva / YAfeH, Yishay / Yosha, Oved (1998): Conflict of Interest in Universal Banking: Bank Lending, Stock Underwriting, and Fund Management. Working Paper, Hebrew University und Tel Aviv University, Juni.

EHRHARDT, OLAF (1997): Börseneinführungen von Aktien am deutschen Kapitalmarkt. Wiesbaden.

Kagel, John H. / Levin, Dan (1986): The Winner's Curse and Public Information in Common Value Auctions. American Economic Review 76, S. 894-920.

Krahnen, Jan P. / Schmid, Frank A. / Theissen, ERIK (1997): Performance and Market Share: Evidence from the German Mutual Fund Industry. Arbeitspapier, Institut für Kapitalmarktforschung - Center for Financial Studies, Frankfurt/Main, September.

Rock, KeVIN (1986): Why New Issues are Underpriced. Journal of Financial Economics 15, S. $187-212$. 